\title{
Ethnobotanical and Ecological Studies of Plants Used in the Treatment of Diabetes in Kwango, Kongo Central and Kinshasa in the Democratic Republic of the Congo
}

\author{
Adrien Tuwisana Masunda ${ }^{1}$, Clement Liyongo Inkoto $^{2}$, Gédéon Ngiala Bongo ${ }^{2}$, \\ Joseph Desiré Oleko Wa Oloko ${ }^{3}$, Koto-Te-Nyiwa Ngbolua ${ }^{2}$, Damien ShaTshibey Tshibangu ${ }^{4}$, \\ Dorothée Dinangayi Tshilanda ${ }^{4}$, Pius Tshimankinda Mpiana ${ }^{4, *}$ \\ ${ }^{1}$ Section of Laboratory Techniques, Higher Institute of Medical Techniques, Kinshasa, Democratic Republic of the Congo
${ }^{2}$ Department of Biology, Faculty of Sciences, University of Kinshasa, Kinshasa, Democratic Republic of the Congo
${ }^{3}$ Higher Institute Superior of Medical Techniques of Tshumbe, Tshumbe, DR Congo
${ }^{4}$ Department of Chemistry, Faculty of Sciences, University of Kinshasa, Kinshasa, Democratic Republic of the Congo \\ Email address: \\ ptmpiana@gmail.com (P. T. Mpiana) \\ ${ }^{*}$ Corresponding author
}

\section{To cite this article:}

Adrien Tuwisana Masunda, Clement Liyongo Inkoto, Gédéon Ngiala Bongo, Joseph Desiré Oleko Wa Oloko, Koto-Te-Nyiwa Ngbolua, Damien ShaTshibey Tshibangu, Dorothée Dinangayi Tshilanda, Pius Tshimankinda. Mpiana. Ethnobotanical and Ecological Studies of Plants Used in the Treatment of Diabetes in Kwango, Kongo Central and Kinshasa in the Democratic Republic of the Congo. International Journal of Diabetes and Endocrinology. Vol. 4, No. 1, 2019, pp. 18-25. doi: 10.11648/j.ijde.20190401.14

Received: December 28, 2018; Accepted: January 31, 2019; Published: February 28, 2019

\begin{abstract}
Non-communicable diseases represent new challenges for the mankind in the fight for health improvement. Among these diseases, diabetes is a major contributor. Diabetes is a serious chronic disease that occurs when the pancreas does not produce enough insulin or when the body is unable to use the insulin produced effectively. This survey was performed in Kwango, Kongo-Central and Kinshasa provinces respectively between October 2016 and September 2017. A questionnaire was administered to the target population in order to collect relevant data. Plant identification was carried out at the herbarium of University of Kinshasa. The findings revealed that the inventoried medicinal flora is made up of 68 species which are divided into 34 families of 58 genera; the leaf is the most commonly used part in the treatment of diabetes while maceration is the most commonly used method of preparation. The use of medicinal plants is reported in almost all age groups from 20-89 years of age but with a predominance among people aged 40-49 years. Trees and phanerophyte species predominate in the flora studied; the majority of users of these medicinal plants have a primary level of education. In-depth phytochemical and pharmacological studies need to be carried out on these plants with a view to their scientific validation in the diabetes management.
\end{abstract}

Keywords: Diabetes, Challenge, Medicinal Plants, Ethnobotany, Kongo Central, Kwango, Kinshasa, DRC

\section{Introduction}

Non-communicable diseases represent new challenges for the mankind in the fight for health improvement. Among these diseases, diabetes is a major contributor because it is considered to be one of the four priority non-communicable diseases targeted for intervention by world leaders $[1,2]$. Diabetes is a serious chronic disease that occurs when the pancreas does not produce enough insulin or when the body is unable to use the insulin produced effectively [3]. However, numerous studies have shown that phytotherapy can be associated with any anti-diabetic treatment and in the case of non-insulino-dependent diabetes, its place may be important as a complement to the diet which has a major role and possible drugs [4]. This is why people are turning to traditional herbal medicine which is less expensive and which they consider as effective as modern therapy. To this end, World Health Organization (WHO) reports that in 
Africa, more than $80 \%$ of the population uses traditional medicine to provide primary health care in both urban and rural areas [5-10].

The Democratic Republic of the Congo (DRC) is a reservoir of both faunal and floristic biodiversity [6-15]. Its flora is full of medicinal plants of biopharmaceutical interest and capable of providing new lead molecules. Thus, ethnopharmacological studies for the scientific validation of these plants are very encouraging, as evidenced by the research results of the last ten years [11-16]. The overall objective of this study was to contribute to the knowledge of the plants used in Kinshasa, Central Kongo and Kwango for the management of diabetes. The specific objectives are to conduct an ethnobotanical survey of the population, to identify and give their ecological characteristics (morphological types, biological types, phytogeographic distributions and biotope) and to highlight their therapeutic virtues.

\section{Material and Methods}

\subsection{Study Area}

The city of Kinshasa is located between $4^{\circ} 18^{\prime}$ and $4^{\circ} 25^{\prime}$ South latitude and between $15^{\circ} 18^{\prime}$ and $4^{\circ} 22^{\prime}$ East longitude. It is bounded to the North and East by Kwango, to the South by Kongo Central and to the West by the Republic of Congo, with an average altitude of $300 \mathrm{~m}$ above the sea level. The city is built on the left bank of the Congo River called the Pool Malebo and is crossed by many rivers called allogenic and the three most important are N'djili, N'sele and MaiNdombe. The climate of Kinshasa city is of the Aw 4 type, i.e. a tropical climate. It is characterized by a large 8-month rainy season (often interspersed with a small dry period straddling between January and February) and between mid-September and mid-May, and a dry season during the rest of the year precisely between mid-May and mid-September [17, 18].

Kwango is located between $6^{\circ} 32^{\prime} 31^{\prime \prime}$ South latitude and $17^{\circ} 24^{\prime \prime}$ East longitude, over an area of $89.974 \mathrm{Km}^{2}$ with a population of 1.994 .036 inhabitants, consisting of Yaka, Suku, Tchokwe, Holo, Lunda Hungana, Mbala, Ngongo tribes. Kwango is divided into five territories namely: Feshi, Kahemba, Kasongo Lunda, Kenge and Popo Kabaka. Kwango is located in a tropical climate zone with two distinct seasons, an 8-month rainy season and a 4-month dry season with a minimum temperature of $20^{\circ} \mathrm{C}$ and a maximum of $27^{\circ} \mathrm{C}$. The vegetation is made up of savannahs, prairies, galleries and forests depending on the territory [19].

Kongo Central is between $4^{\circ}$ and $6^{\circ}$ south latitude and 12 and $16^{\circ}$ East longitude over a $53920 \mathrm{~km}^{2}$ area. With a population of 3615504 inhabitants, there are the Yombe, Nianga, Ndibu peoples. The population is unevenly distributed in space. Matadi, the chief town of this province, represents 7\%, so much so that the Bas Fleuve and Cataractes represent $28 \%$ and $36 \%$ respectively of the population. Boma $10 \%$ and Lukaya $2.3 \%$ and the other populations are found in villages and chiefdoms. The climate in Kongo Central is of a tropical Sudanese type climate, subdivided into two types: a first one that extends along the coast, is a steppe type climate with very high variability that lasts for months (from May 15 to September 15) while the other tropical climate that lasts 8 months. Bas-Fleuve is an area where the risk of drought is very high. An ecological niche of Mangrove is observed off the coast. The minimum temperature can be between $15^{\circ} \mathrm{C}$ and $17^{\circ} \mathrm{C}$ in the coastal zone and then the maximum at $27^{\circ} \mathrm{C}$, in other territories the minimum $20^{\circ} \mathrm{C}$ and maximum $27^{\circ} \mathrm{C}$ [19].

\subsection{Methodology}

An ethnobotanical study was carried out between October 2016 and September 2017 using a survey approach mainly among traditional medicine stakeholders who market their products in different markets of these aforementioned provinces, of which 68 people were interviewed directly using a predefined survey form.

The identification of plants was carried out at the herbarium of the Department of Biology, Faculty of Science, University of Kinshasa by comparison with the available specimens and/or the herbarium of the Jardin Botanique de Kisantu.

At part from the questionnaire used, the ethnobotanical data collected were then supplemented by information on ecological types as follows: Morphological types: Tree (T), Shrub (Sh), Annual grass (AG), Perennial grass (Pg), Liana (Li) and Rhizome (Rh) while the Biological types were: Bulbous geophyte (Gbu), Rhizomant geophyte (Grh), Tuberous geophyte (Gtu), Mesophanerophyte (Msph), Megaphanerophyte (Mgph), Microphanerophyte (Mcph), Nanophanerophyte (Nph), Lying Phanerophyte (Lph), Upright Phanophyte (Thd), Climbing Theophyte; Phytogeographic distribution: pantropical (Pan), afrotropical, Sarcochores (Sarc), guinea-congolian (GC), Balochores (Bal), Palio-tropical (Pal), pterochores (Pte), cosmopolitan (HC) and sclerochorous (Scl), Lower Guinea (BG), Central Guinea-Congolian (CGC), African-American (Aa), Lower Guinea-Congolian (BGC), Guinean and Zambézian (GS-Z) and Biotope Type: Crop (Crop), Forest (Fo), Savannah (Sa), Ruderal (Rud), Parasite (Para).

\subsection{Data Analysis}

The data collected in the field were analyzed using SPSS software version 20.0 for descriptive statistics.

\section{Results}

The ethnobotanical data and the ecological characters of listed plants are presented in the table below. 
Table 1. Ethnobotanical data and ecological characters of listed plants.

\begin{tabular}{|c|c|c|c|c|c|c|c|c|}
\hline \multirow{2}{*}{$\mathbf{N}^{\circ}$} & \multirow{2}{*}{ Scientific Names (Famillies) } & \multirow{2}{*}{ Local names } & \multirow{2}{*}{$\begin{array}{l}\text { Used } \\
\text { parts }\end{array}$} & \multirow{2}{*}{$\begin{array}{l}\text { Mode of } \\
\text { preparation }\end{array}$} & \multicolumn{4}{|c|}{ Ecological characteristics } \\
\hline & & & & & M.T & B.T & P.D & Biotope \\
\hline 01 & $\begin{array}{l}\text { Albizia adianthifolia (Schum) W.Wight } \\
\text { (Leguminosae) }\end{array}$ & Sela & Leaves & Infusion & $\mathrm{T}$ & Msph & At & $\mathrm{F}$ \\
\hline 02 & Allium cepa L. (Amaryllidaceae) & Litungulu & Bulb & Maceration & PG & $\mathrm{Gb}$ & Paléo & Cult \\
\hline 03 & Allium sativum L. (Amaryllidaceae) & Aïl & Bulb & Maceration & PG & $\mathrm{Gb}$ & Paléo & Cult \\
\hline 04 & $\begin{array}{l}\text { Alchornea cordifolia (Schumach. \& Thonn.) } \\
\text { Müll.Arg. (Euphorbiaceae) }\end{array}$ & Liboto & Leaves & Decoction & Shrub & Mcph & At & $\mathrm{F}$ \\
\hline 05 & Mangifera indica L. (Anacardiaceae) & Manga & Leaves & Decoction & $\mathrm{T}$ & Msph & Pan & Cult \\
\hline 06 & Spondias mombin L. (Anacardiaceae) & Mingenge & Leaves & Decoction & $\mathrm{T}$ & Msph & Pan & $\mathrm{F}$ \\
\hline 07 & Annona senegalensis Pers. (Annonaceae) & Mulolo & Bark & Decoction & Shrub & Mcph & GC & $\mathrm{F}$ \\
\hline 08 & $\begin{array}{l}\text { Xylopia aethiopica (Dunal) A.Rich. } \\
\text { (Annonaceae) }\end{array}$ & Nsombo & Bark & Decoction & $\mathrm{T}$ & Mgph & At & $\mathrm{F}$ \\
\hline 09 & Catharanthus roseus (L.) G.Don (Apocynaceae) & Kilungu & Leaves & Decoction & PG & Chdr & Sarco & Cult \\
\hline 10 & Rauvolfia vomitoria Afzel. (Apocynaceae) & Isumbubululu & Bark & Decoction & Shrub & Mcph & Méso & $\mathrm{F}$ \\
\hline 11 & Raphia gentiliana De Wild. (Arecaceae) & Bankulu & Fruit & Decoction & $\mathrm{T}$ & Msph & Sarco & Cult \\
\hline 12 & Calendula officinalis L. (Compositae) & Mundudi ndudi & Bark & Decoction & AG & Thdr & Bal & Cosmo \\
\hline 13 & Vernonia amygdalina Delile (Compositae) & Nkulu nkasi & $\begin{array}{l}\text { Bark } \\
\text { Leaves }\end{array}$ & Decoction & Shrub & Mcph & At & $\mathrm{Fa}$ \\
\hline 14 & $\begin{array}{l}\text { Gymnanthemum coloratum (Willd.) H.Rob. \& } \\
\text { B.Kahn }\end{array}$ & Kilulukunju & $\begin{array}{l}\text { Bark, } \\
\text { Leaves }\end{array}$ & Decoction & Shrub & Msph & Pan & $\mathrm{F}$ \\
\hline 15 & Betula pendula Roth (Betulaceae) & - & Leaves & Infusion & $\mathrm{T}$ & Msph & Sarco & Cult \\
\hline 16 & Brassica oleracea L. (Brassicaceae) & Choux & Leaves & Decoction & $\mathrm{AG}$ & Thdr & Bal & Cult \\
\hline 17 & Carica papaya L. (Caricaceae) & Payipayi & Leaves & Infusion & A & Msph & Pan & Cult \\
\hline 18 & Senna alata (L.) Roxb. (Leguminosae ) & Mbau mbau & Leaves & Decoction & Shrub & $\mathrm{Nph}$ & Pan & Rud \\
\hline 19 & $\begin{array}{l}\text { Senna timoriensis (DC.) H.S.Irwin \& Barneby } \\
\text { (Leguminosae) }\end{array}$ & Mapalata & Leaves & Decoction & $\mathrm{T}$ & $\mathrm{Nph}$ & Bal & Rud \\
\hline 20 & $\begin{array}{l}\text { Dysphania ambrosioides (L.) Mosyakin \& } \\
\text { Clemants (Amaranthaceae) }\end{array}$ & Nkasi kindongo & Bark & Maceration & AG & Thd & $\mathrm{Scl}$ & Cult \\
\hline 21 & Terminalia chebula Retz. (Combretaceae) & Madame & Leaves & Infusion & $\mathrm{T}$ & $\mathrm{McPh}$ & Paléo & Cult \\
\hline 22 & Costus phyllocephalus K.Schum. (Costaceae) & Minkeni & $\begin{array}{l}\text { Leaves, } \\
\text { Root }\end{array}$ & Decoction & PG & Grh & $\mathrm{GC}$ & $\mathrm{F}$ \\
\hline 23 & Momordica charantia L. (Cucurbitaceae) & Lumbusu & $\begin{array}{l}\text { Leaves, } \\
\text { fruit }\end{array}$ & Infusion & PG & Thgr & $\mathrm{GC}$ & Cult \\
\hline 24 & Dioscorea praehensilis Benth. (Dioscoreaceae) & Bandindi & Leaves & Decoction & $\mathrm{L}$ & Ggr & Pte & $\mathrm{F}$ \\
\hline 25 & Bridelia ferruginea Benth. (Phyllanthaceae) & Kimuindu & Root & Decoction & $\mathrm{T}$ & Mcph & At & Sav. \\
\hline 26 & Maprounea africana Müll.Arg. (Euphorbiaceae) & Kiseyi kiseyi & Leaves & Infusion & Shrub & $\mathrm{MsPh}$ & AT & $\mathrm{F}$ \\
\hline 27 & Caesalpinia bonduc (L.) Roxb. (Leguminosae) & - & Leaves & Decoction & $\mathrm{T}$ & Phgr & Bal & Cult \\
\hline 28 & $\begin{array}{l}\text { Cyamopsis tetragonoloba (L.) Taub. } \\
\text { (Leguminosae) }\end{array}$ & - & Leaves & Decoction & AG & chdr & Pan & Cult \\
\hline 29 & Erythrina abyssinica DC.(Leguminosae) & Kikumbu & Leaves & Decoction & $\mathrm{T}$ & Msph & Bal & Sav \\
\hline 30 & Pterocarpus marsupium Roxb. (Leguminosae) & Nkila & Leaves & Decoction & $\mathrm{T}$ & Msph & Pte & $\mathrm{F}$ \\
\hline 31 & Pterocarpus angolensis DC. (Leguminosae) & Nkula & Seed & Decoction & $\mathrm{T}$ & Msph & At & $\mathrm{F}$ \\
\hline 32 & Trigonella foenum-graecum L. (Leguminosae) & Kiwaya & Leaves & Maceration & $\mathrm{AG}$ & Thdr & Pan & Cult \\
\hline 33 & Scorodophloeus zenkeri Harms (Leguminosae) & Kiwaya & Leaves & Decoction & $\mathrm{T}$ & $\mathrm{MsPh}$ & BG & $\mathrm{F}$ \\
\hline 34 & Phaseolus vulgaris L. (Leguminosae) & Haricot & $\begin{array}{l}\text { Leaves, } \\
\text { Bark }\end{array}$ & Decoction & AG & Chgr & $\operatorname{Cos}$ & Cult \\
\hline 35 & Gnetum africanum Welw. (Gnetaceae). & Mfumbwa & Leaves & Decoction & PG & Phgr & CGC & $\mathrm{F}$ \\
\hline 36 & Ocimum gratissimum L. (Lamiaceae) & $\begin{array}{l}\text { Dinsunsu } \\
\text { nsusudinene }\end{array}$ & Leaves & Infusion & Shrub & Chd & Paléo & Sav. \\
\hline 37 & Ocimum minimum L. (Lamiaceae) & $\begin{array}{l}\text { Dinsunsu nsusu } \\
\text { difioti }\end{array}$ & Leaves & Infusion & $\mathrm{AG}$ & Chd & $\mathrm{Scl}$ & Cult \\
\hline 38 & Persea americana Mill. (Lauraceae) & Divoka & Leaves & Decoction & $\mathrm{T}$ & Msph & Pan & F, Cult \\
\hline 49 & Viscum album L. (Santalaceae) & - & Leaves & Infusion & Shrub & Thd & Sarco & Para \\
\hline 40 & Punica granatum L. (Lythraceae) & - & Flower & Infusion & $\mathrm{T}$ & Msph & Sarco & Cult \\
\hline 41 & $\begin{array}{l}\text { Abelmoschus esculentus (L.) Moench } \\
\text { (Malvaceae) }\end{array}$ & Dongo dongo & Fruit & Maceration & AG & Thd & Pan & Cult \\
\hline 42 & Urena lobata L. (Malvaceae) & Pungala & $\begin{array}{l}\text { Leaves, } \\
\text { root }\end{array}$ & Decoction & PG & $\mathrm{NPh}$ & Pan & Cult \\
\hline 43 & Azadirachta indica A.Juss. (Meliaceae) & Nime & Leaves & Decoction & $\mathrm{T}$ & Mcph & Sarco & Cult \\
\hline 44 & Ficus benghalensis L. (Moraceae) & Nsanda & $\begin{array}{l}\text { leaves, } \\
\text { bark }\end{array}$ & Infusion & $\mathrm{T}$ & Mcph & Sarco & Cult \\
\hline 45 & Moringa oleifera Lam. (Moringaceae) & Moringa & Leaves & Decoction & $\mathrm{T}$ & Msph & Pan & Cult \\
\hline 46 & Eucalyptus globulus Labill. (Myrtaceae) & Bikalubitus & Leaves & Infusion & $\mathrm{T}$ & Msph & Sarco & Cult \\
\hline 47 & Psidium guajava L. (Myrtaceae) & Mapela & Leaves & Decoction & $\mathrm{T}$ & $\mathrm{MsPh}$ & Pan & Cult \\
\hline 48 & Syzygium cumini (L.) Skeels (Myrtaceae) & Telezia & Seed & Infusion & $\mathrm{T}$ & $\mathrm{MsPh}$ & Sarco & Cult \\
\hline 49 & Olea europaea subsp. cuspidata (Wall. \& G.Don) & Olivier & Leaves & Infusion & $\mathrm{T}$ & Msph & Sarco & Cult \\
\hline
\end{tabular}




\begin{tabular}{|c|c|c|c|c|c|c|c|c|}
\hline \multirow{2}{*}{$\mathbf{N}^{\circ}$} & \multirow{2}{*}{ Scientific Names (Famillies) } & \multirow{2}{*}{ Local names } & \multirow{2}{*}{$\begin{array}{l}\text { Used } \\
\text { parts }\end{array}$} & \multirow{2}{*}{$\begin{array}{l}\text { Mode of } \\
\text { preparation }\end{array}$} & \multicolumn{4}{|c|}{ Ecological characteristics } \\
\hline & & & & & M.T & B.T & P.D & Biotope \\
\hline 50 & $\begin{array}{l}\text { Cif. (Oleaceae). } \\
\text { Millettia eetveldeana (Micheli) Hauman } \\
\text { (Leguminosae) }\end{array}$ & Mbwenge & Root & Decoction & $\mathrm{T}$ & Msph & Ballo & $\mathrm{F}$ \\
\hline 51 & Millettia laurentii De Wild. (Leguminosae) & Kiboto & Bark & Decoction & $\mathrm{T}$ & Msph & BGC & $\mathrm{F}$ \\
\hline 52 & Sesamum indicum L. (Pedaliaceae) & Wangila & Seed & Decoction & $\mathrm{AG}$ & Thd & GC & Cult \\
\hline 53 & $\begin{array}{l}\text { Phyllanthus amarus Schumach. \& } \\
\text { Thonn.(Phyllanthaceae) }\end{array}$ & - & Leaves & Infusion & PG & $\mathrm{NPh}$ & Pal & Rud \\
\hline 54 & Phyllanthus niruri L. (Phyllanthaceae) & - & Seed & Infusion & PG & Chdr & $\mathrm{Bal}$ & Rud \\
\hline 55 & Cymbopogon citratus (DC.) Stapf (Poaceae) & Sinda & Leaves & Infusion & PG & Hcès & AT & Cult \\
\hline 56 & $\begin{array}{l}\text { Cymbopogon densiflorus (Steud.) Stapf } \\
\text { (Poaceae) }\end{array}$ & Lusangu sangu & Leaves & Infusion & PG & Hcès & $\mathrm{Hc}$ & Cult \\
\hline 57 & Oryza sativa L. (Poaceae). & Loso & Leaves & Decoction & PG & Thd & Pan & Cult \\
\hline 58 & Polygala acicularis Oliv. (Polygalaceae) & Lunsambi nsambi & Leaves & Decoction & Shrub & $\mathrm{NPh}$ & AT & Cult \\
\hline 59 & Morinda lucida Benth (Rubiaceae) & Nsiki & Leaves & Decoction & $\mathrm{T}$ & Msph & $\mathrm{GC}$ & $\mathrm{PF}$ \\
\hline 60 & $\begin{array}{l}\text { Morinda morindoides (Baker) Milne-Redh. } \\
\text { (Rubiaceae) }\end{array}$ & Kongobololo & Leaves & Decoction & $\mathrm{L}$ & Lph & At & SF \\
\hline 61 & $\begin{array}{l}\text { Sarcocephalus pobeguinii Hua ex Pobég. } \\
\text { (Rubiaceae) }\end{array}$ & Kenga kimansa & Leaves & Decoction & $\mathrm{T}$ & Mcph & Sarco & $\mathrm{W}$ \\
\hline 62 & $\begin{array}{l}\text { Sarcocephalus latifolius (Sm.) E.A.Bruce } \\
\text { (Rubiaceae) }\end{array}$ & Kilolo & Leaves & Decoction & $\mathrm{T}$ & $\mathrm{MsPh}$ & $\mathrm{GC}$ & $\mathrm{F}$ \\
\hline 63 & Solanum aethiopicum L. (Solanaceae) & Solo & Fruit & Decoction & $\mathrm{AG}$ & Thdr & At & Cult \\
\hline 64 & Solanum melongena L. (Solanaceae) & Mbolongo & Fruit & Decoction & $\mathrm{AG}$ & Thdr & Pan & Cult \\
\hline 65 & Schwenckia americana L. (Solanaceae). & Ntumpu, dinkombo & Leaves & Decoction & AG & Chdr & Аa & Sav \\
\hline 66 & $\begin{array}{l}\text { Cola acuminata (P.Beauv.) Schott \& } \\
\text { Endl.(Malvaceae) }\end{array}$ & Makasu & Leaves & Decoction & $\mathrm{T}$ & Msph & $\mathrm{GC}$ & $\mathrm{PF}$ \\
\hline 67 & Lippia multiflora Moldenke (Verbenaceae) & Bulukutu & Leaves & Infusion & $\mathrm{T}$ & Chdr & AT & Sav \\
\hline 68 & Vitex madiensis Oliv. (Lamiaceae) & Kifilu & $\begin{array}{l}\text { Root, } \\
\text { Leaves }\end{array}$ & Infusion & $\mathrm{T}$ & $\mathrm{MsPh}$ & GSZ & Sav \\
\hline
\end{tabular}

Legend: M. T: Morphological types, B. T: Biological types, P. D: Phytogeographic distribution, Tree (T), Annual grass (AG), Perennial grass (PG), Liana (L), Rhizome (Rh), Bulbous geophyte (Gbu), Rhizomant geophyte (Grh), Tuberous geophyte (Gtu), Mesophanerophyte (Msph), Megaphanerophyte (Mgph), Microphanerophyte (Mcph), Nanophanerophyte (Nph), Lying Phanerophyte (Lph), Upright Phanophyte (Thd), Climbing Theophyte, Pantropical (Pan), afrotropical, Sarcochores (Sarc), guinea-congolian (GC), Balochores (Bal), Palio-tropical (Pal), pterochores (Pte), cosmopolitan (HC), Sclerochorous (Scl), Lower Guinea (BG), Central Guinea-Congolian (CGC), African-American (Aa), Lower Guinea-Congolian (BGC), Guinean and Zambézian (GS-Z), Crop (Crop), Forest (F), Savannah (Sa), Ruderal (Rud), Parasite (Para), Cosmopolite (Cosmo), Fallow (Fa), Primary Forest (PF), Secondary Forest (SF), Woodland (W).

\section{Discussion}

The different families of listed plants are presented in figure

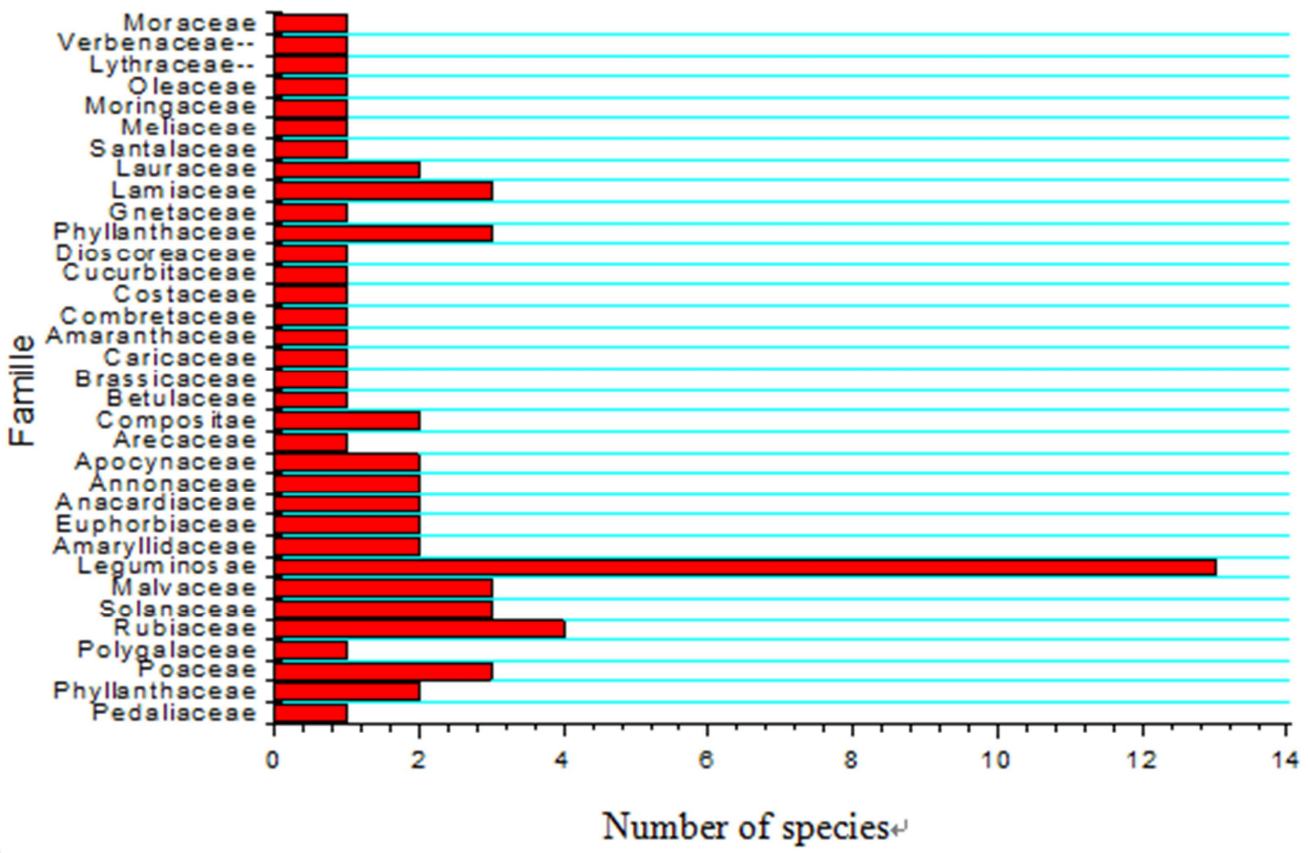

Figure 1. Different families of inventoried plants. 
In total, 34 families were listed, and of these families, the Leguminosae has $38.2 \%$ of species of this area, followed by Rubiaceae (11.76\%), Poaceae, Solanaceae, Malvaceae, Phyllanthaceae, and Lamiaceae $(8.82 \%$ each) respectively. These results are similar to recent data indicating that Leguminosae is one of the six most represented angiosperms families [20].

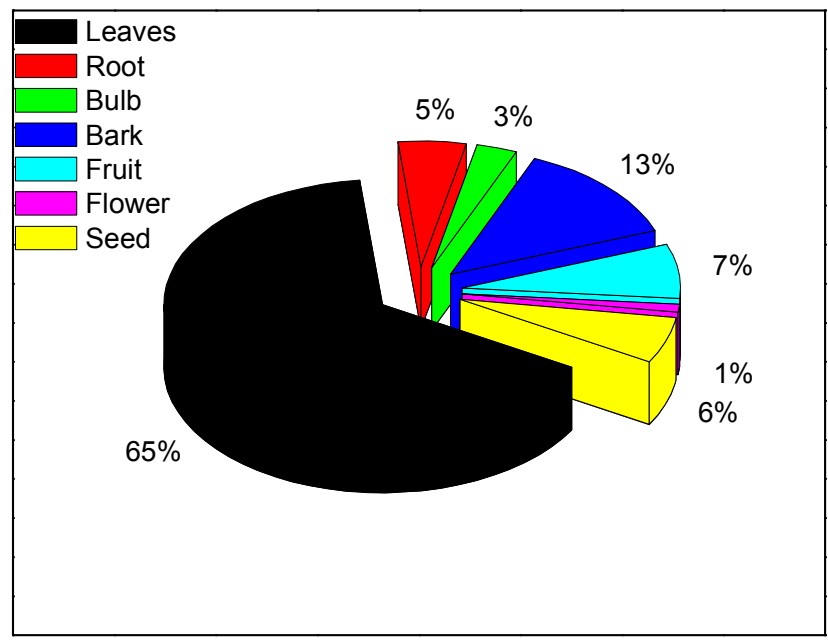

Figure 2. Different parts used.

From the investigation, it was shown that the leaves are the most commonly used part in the treatment of diabetes. The bark was indicated at the second position (13\%) followed by fruit $(7 \%)$, seed $(6 \%)$ and root $(5 \%)$ respectively. At last, it was observed that flower and bulb are the least used. These findings are similar to Ndjouondo et al. [21] who reported that the leaves were the most commonly used part in traditional medicine. On the other side, others researchers reported that the root was much used $[10,18]$.

The different modes of recipes preparation are presented in the figure below.

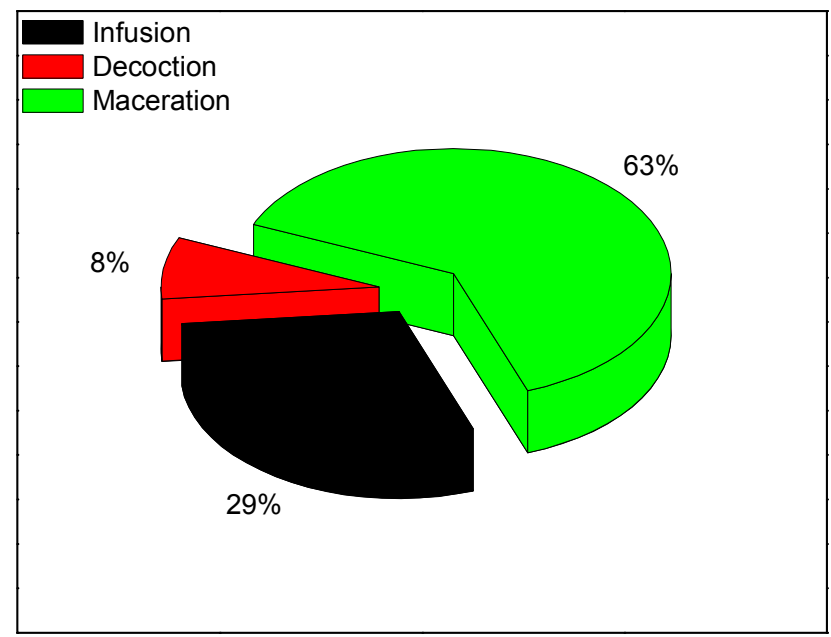

Figure 3. Different modes of recipes preparation.

As demonstrated above, three different modes for recipe preparation of medicinal plants were reported. The maceration $(63 \%)$ was reported as the most commonly used method, followed by the infusion (29\%) and decoction (8\%).

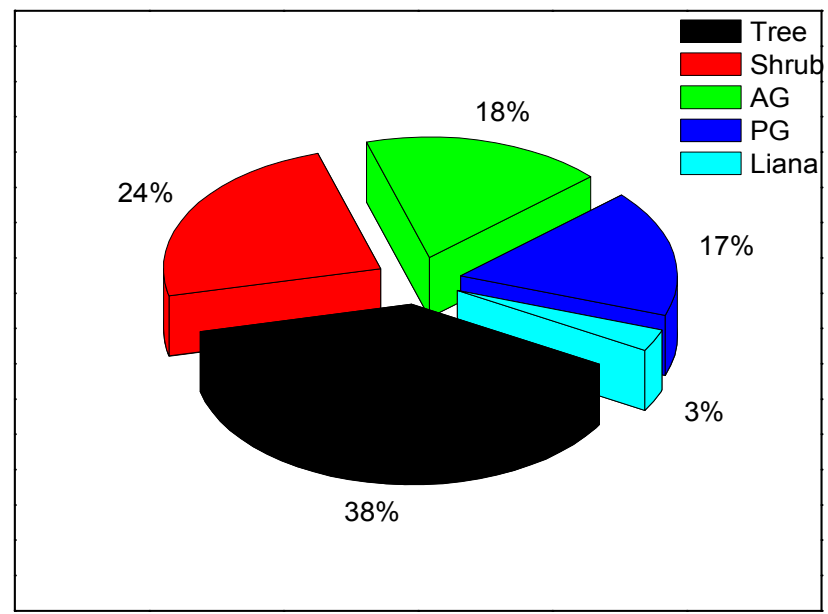

Figure 4. Morphological types of listed plants.

Trees present a higher value $(38 \%)$, followed by shrubs (24\%), annual grass and perennial grass (18\% each) knowing that liana species are less represented in this study area. This predominance of trees shows that this ecosystem is more species-diversified than other non-forest ecosystems [10, 22].

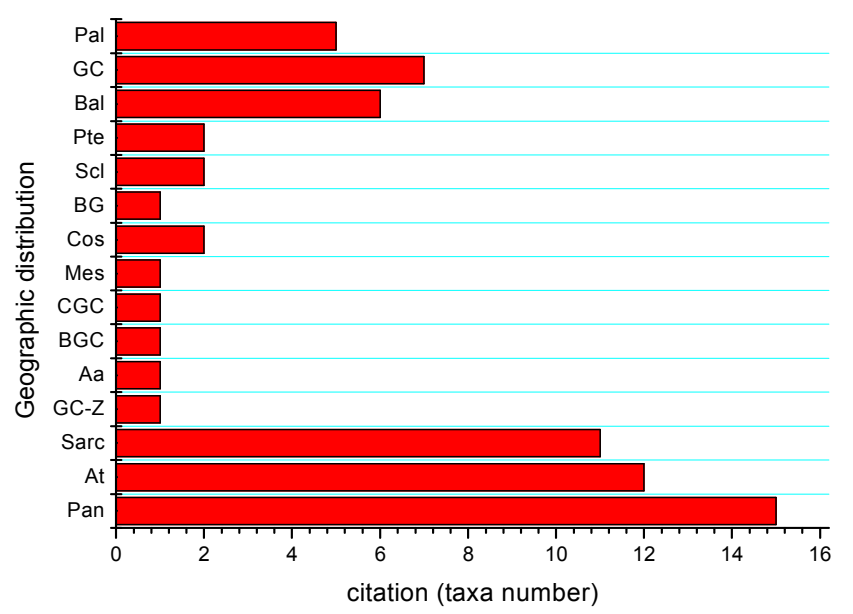

Figure 5. Phytogeographical distribution of plants.

Figure 5 shows that the listed species are widely distributed throughout the world. We observe a predominance of panetropical species (Pan: 23\%), followed by afro-tropical species (At: 19\%), Sarcochores (Sarc: 17\%), guinea-congolian (GC: 10\%), Balochores (Bal: 9\%), Paliotropical (Pal: 7\%), pterochores (Pte), cosmopolitans (HC) and sclerochores (Scl) 3\% each. While Lower Guinea (BG), Central Guinea-Congolian (CGC), African Americans (Aa), Lower Guinea-Congolian (BGC), Guinea-Congolian and Zambezians (GS-Z) each represent only 1\%. These results show that these plant taxa are widely distributed in Africa. Thus, their protection should be a concerted effort at the national, sub-regional and regional levels, based on a certain political will. 
The biological types of plants are presented in the figure below.

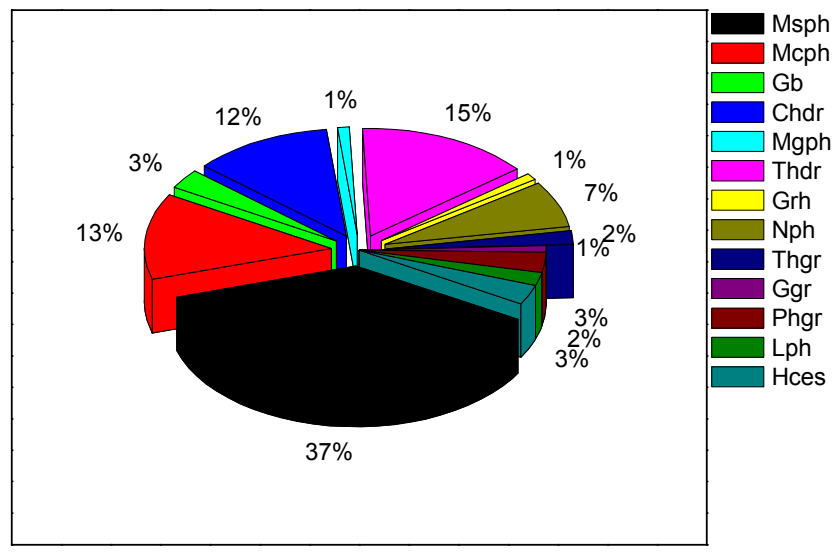

Figure 6. Biological types of plants.

It appears that the antidiabetic plants listed belong to 13 biological types, the most common of which are mesophanerophytes (Msph: 37\%), followed by therophytes (Thdr: 15), microphanerophytes (Mcph: 13\%), erect camphytes (Chdr: 12\%), nanophanerophyte (Nph: 7\%), bulbous geophytes $(\mathrm{Gb})$, climbing phanerophytes (Phgr) and cespiteux grasses (Hces) each account for 3\%. While megaphanerophytes (Mgph), rhizomatous geophytes (Grh), climbing geophytes (Ggr) and vine phanerophytes are almost at the trace state $(1 \%$ each).

The predominance of phanerophytes among listed taxa is a characteristic of tropical regions and may also correlate with the fact that their tissues have been claimed to synthesize secondary bioactive metabolites [10, 23-24].

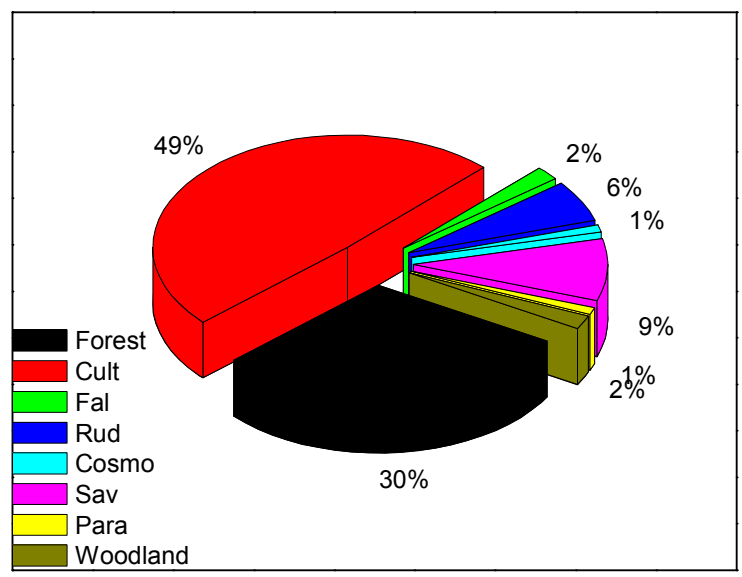

Figure 7. Types of biotopes of listed plants.

The biotope type of the inventoried plant species has been classified as follows: forest, savannah, ruderal habitat, fallow, woodland, parasitic plants, cultivated plants and cosmopolitan plants. About $49.3 \%$ are cultivated plants followed by forest $(30.4 \%)$, savannah $(8.7 \%)$ and ruderal $(5.8 \%)$ plants. While fallow, cosmopolitan, parasitic and undergrowth plants represent $1.45 \%$ each. This predominance of the cultivated type can be justified by the fact that the population seeks to cultivate various useful plants close to their habitations.

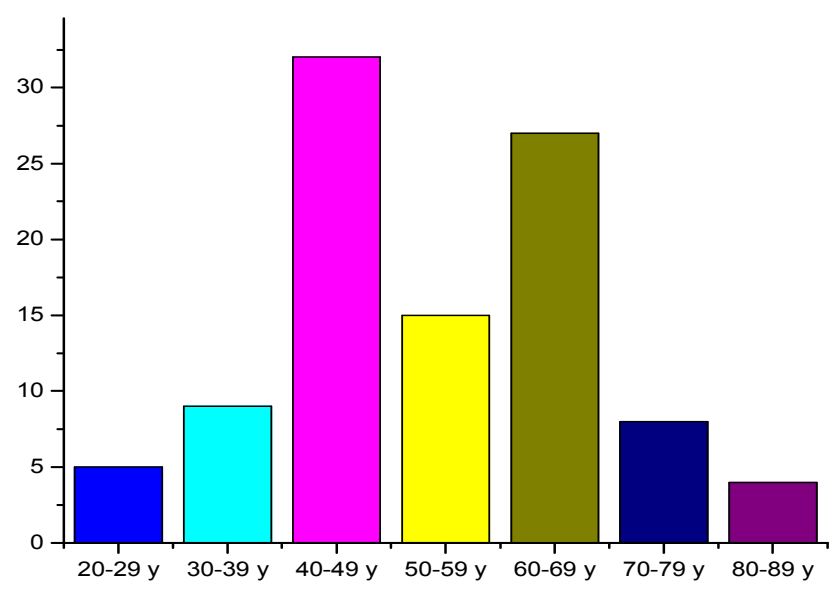

Figure 8. Distribution of respondents according to age.

This figure shows that the use of medicinal plants is almost universal but concentrated between 40-49 years (32\%), followed by the age group 60-69 years. Intakes for the elderly (20-39 years) are the least common.

The elderly are mostly heads of households and represent family authority. Mulwele et al. [25], who inventoried medicinal plants marketed in the various markets of Kwango, reported that 60 out of the 75 respondents were over 40 years of age. These elderly people are also expected to provide more reliable information, as they hold much of the ancestral knowledge that is part of the oral tradition [26]. Anyinam [27] shows that knowledge of the properties and uses of medicinal plants is acquired through a long experience accumulated and transmitted from one generation to the next. Benkhnigue et al. [28] support this view by showing that experience with age is the main source of information at the local level about the use of plants in traditional medicine.

The distribution of respondents according to sex (gender) is presented in the figure below.

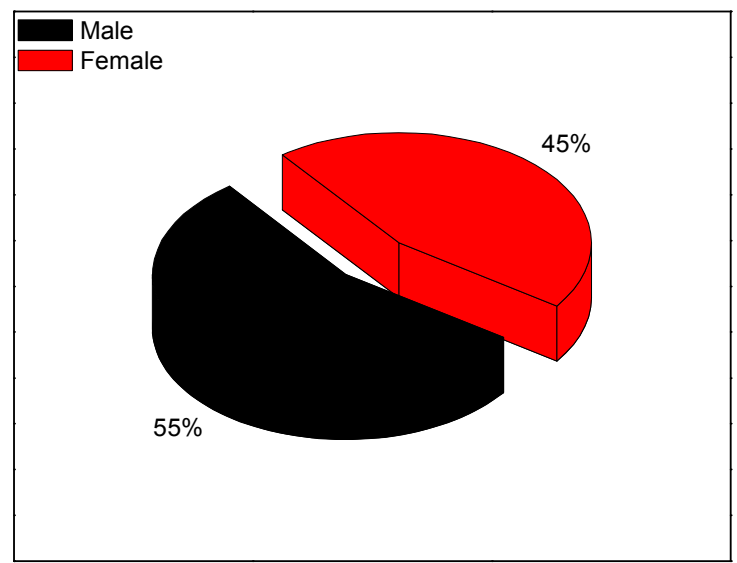

Figure 9. Distribution of respondents according to gender.

From the above figure, it was observed that $545 \%$ were male and $45 \%$ were female. Mulwele et al. [25] reported that female use medicinal plants much more in Douala, Cameroon. 
The figure below presents the distribution of respondents according to their education level.

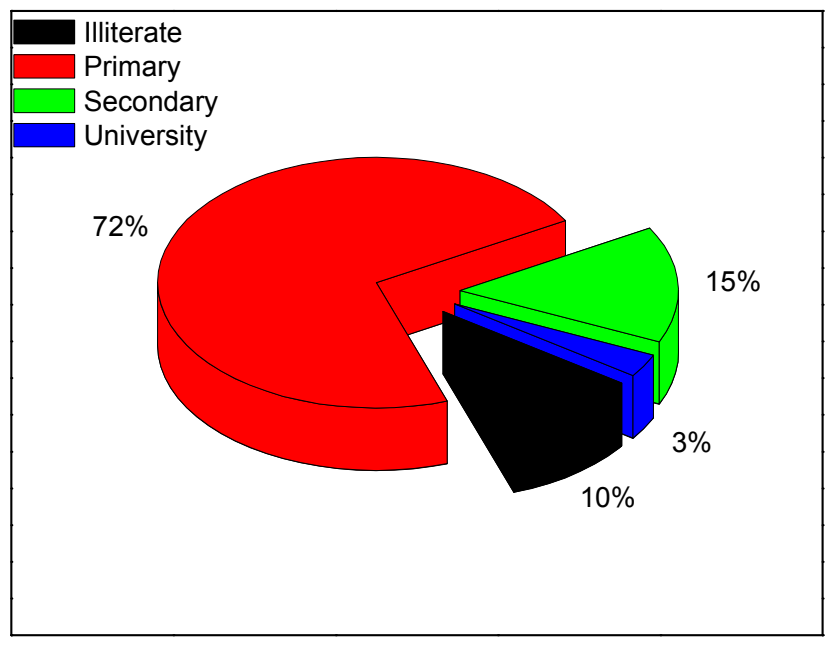

Figure 10. Distribution of respondents according education level.

The above figure showed that $72 \%$ of medicinal plant users have been enrolled in primary school, 15\% have reached secondary school and 3\% have reached university level. There were about $10 \%$ of people with no education at the school level. These results corroborate with the work of Mulwele et al. [25] and Kahouadji [29] showed that riparian surveyed in Douala have a level of education at least equivalent to that of primary school.

\section{Conclusion}

The purpose of this study was to identify the anti-diabetic medicinal plants used in Kinshasa, Kongo Central and Kwango provinces.

The results obtained in this study show that: The inventoried medicinal flora is made up of 68 species which are divided into 34 families of 58 genera; the leaf is the most commonly used part in the treatment of diabetes $(65.3 \%)$ and maceration is the most commonly used method of preparation; the use of medicinal plants is reported in almost all age groups from 20-89 years of age but with a predominance among people aged 40-49 years; Trees and phanerophyte species predominate in the flora studied; the majority of users of these medicinal plants have a primary level of education.

It would be desirable that in-depth phytochemical and pharmacological studies be carried out on these plants with a view to their scientific validation.

\section{References}

[1] Alwan A., Maclean DR., Riley LM., Espaignet ET., Mathers CD., Stevens GA. (2010). Monitoring and surveillance of chronic non-communicable diseases: progress and capacity in high-burden countries. Lancet.; 376 (9755): 1861-8.

[2] Bourne RR., Stevens GA., White RA., Smith JL., Flaxman
SR., Price H. (2013). Causes of vision loss worldwide, 19902010: a systematic analysis; Lancet Global Health 1: 339349.

[3] Olaokun OO., McGaw LJ., Awouafack MD., Eloff JN., Naidoo V. (2014). The potential role of GLUT4 transporters and insulin receptors in the hypoglycaemic activity of Ficus lutea acetone leaf extract. BMC Complement Altern Med.; 14: 269.

[4] Zhou L., Zhoci S., Tang J., Zhang K., Guang L., HoungY., Xu Y., Ying Y., Zang I., Li D. (2009). Protective effect of berberin on beat cells in streptozotocin. and carbohydrate fat diet induced diabetic rats. European journal of pharmacology 606: 262-268.

[5] World Health Organization. Traditional medicine strategy 2002-2005, 2002. http: //www.who.int/medicines/library/trm trat eng.pdf.

[6] Ngbolua KN, Rafatro H., Rakotoarimanana H., Urverg RS., Mudogo V., Mpiana PT., Tshibangu DST. (2011). Pharmacological screening of some traditionally-used antimalarial plants from the Democratic Republic of Congo compared to its ecological taxonomic equivalence in Madagascar. Int. J. Biol. Chem. Sci., Vol. 5, no. 5, pp. 17971804,2011

[7] Inkoto LC., Bongo NG., Kapepula MP., Masengo AC., Gbolo ZB., Tshiama C., Ngombe KB., Iteku BJ., Mbemba FT, Mpiana PT., Ngbolua KN. (2018). Microscopic features and chromatographic fingerprints of selected congolese medicinal plants: Aframomum alboviolaceum (Ridley) K. Schum, Annona senegalensis Pers. and Mondia whitei (Hook.f.) Skeels. Emergent Life Sciences Research, 4 (1): 1-10.

[8] Ngbolua KN., Inkoto CL., Bongo GN., Lufuluabo GL., Kutshi NN., Masengo CA. Santos Kavumbu SM., Gbolo BZ., Tshilanda DD., Mpiana PT. (2018). Microscopy features, Phytochemistry and Bioactivity of Mondia whitei L. (Hook F.) (Apocynaceae): A mini-review. Discovery Phytomedicine 5(3): 34-42.

[9] Ngbolua KN., Inkoto CL., Bongo GN, Lengbiye EM., Lufuluabo GL., Masengo CA., Tshibangu DST., Tshilanda DD. and Mpiana PT. (2018). Phytochemistry and Bioactivity of Annona reticulata L. (Annonaceae): A Mini-review. SARJNP; 1 (1): 1-11.

[10] Ngbolua KN., Shetonde OM., Inkoto CL., Masengo CA., Tshibangu DST., Gbolo BZ., Robijaona B., Fatiany PR. and Mpiana PT. (2016). Ethno-botanical survey of plant species used in traditional medicine in Kinshasa city (Democratic Republic of the Congo), Tropical Plant Research 2016; 3 (2): 413 - 427.

[11] Ngbolua KN., Bishola TT., P.T. Mpiana, V. Mudogo, D.S.T. Tshibangu, K.N. Ngombe, E.G. Ekutsu, D.D. Tshilanda, Z.B. Gbolo, T.D. Mwanangombo, P.R. Fatiany, R. Baholy. Ethnobotanical survey, in vitro antisickling and free radical scavenging activities of Garcinia punctata Oliv. (Clusiaceae). Journal of Advanced Botany \& Zoology V1I2, 2014e. DOI: 10.15297/JABZ. V1I2.04.

[12] G. E. Ekutsu, K. N. Ngbolua, B. M. Bolaa, P. T. Mpiana, B. P. Ngoy, C. A. Masengo, G. N. Bongo. Enquête sur la pharmacopée des bonobos (Pan paniscus, Primates) dans un foyer endémique et Mise en évidence de l'activité antidrépanocytaire chez un taxon végétal (Treculia africana Decne ex Trécul, Moraceae) testé in vitro. International Journal of Innovation and Applied Studies Vol. 14, no. 2, pp. 315-326, 2016. 
[13] Ngbolua KN., Bolaa BM., Mpiana PT., Ekutsu EG., Masengo AC, Tshibangu DST., Mudogo V., Tshilanda DD., Kowozogono KR. (2015). Great Apes Plant Foods as Valuable Alternative of Traditional Medicine in Congo Basin: The Case of Non- Human Primate Bonobos (Pan paniscus) Diet at Lomako Fauna Reserve, Democratic Republic of the Congo. J. of Advanced Botany and Zoology, V3I1, DOI: 10.15297/JABZ. V3I1.01.

[14] Ilumbe BG., Damme PV., F.L. Lukoki, V. Joiris, M. Visser, J. Lejoly. Contribution à l'étude des plantes médicinales dans le traitement des hémorroïdes par les pygmées de Twa et leur voisin Oto de bikoro, RDC. Congo Sciences Vol. 2, no. 1, pp. 46-54, 2014.

[15] Guohua H., Yanhua L., Rengang M., Dongzhi W., Zhengzhi M., Hua Z. (2009). Aphrodisiac properties of Allium tuberosum seeds extract Journal of Ethnopharmacology 122 (3): $579-582$

[16] Kabena NO., Ngombe KN., Ngbolua KN., Kikufi BA., Lassa L., Mboloko E., Mpiana PT., Lukoki FL. (2014). Etudes ethnobotanique et écologique des plantes d'hygiène intime féminine utilisées à Kinshasa (R. D Congo). Int J. Biol. Chem.Sci. 8 (6):2626-2642.

[17] Lassa KL. (2007). Inventaire préliminaire des plantes médicinales vendues dans quelques marchés de la partie Est de la ville de Kinshasa: cas de Mont - Amba et Tshangu. Mémoire de DEA, Faculté des Sciences, Université de Kinshasa. p67.

[18] Ngbolua KN., Mandjo BL., Munsebi JM., Ashande MC., Lengbiye EM., Lionel S. Asamboa, Konda RK., Dianzuangani DL., Ilumbe M., Nzudjom AB., Mukebayi K. and Mpiana PT. (2016). Etudes ethnobotanique et écologique des plantes utilisées en médecine traditionnelle dans le District de la Lukunga à Kinshasa (RD du Congo) International Journal of Innovation and Scientific Research, 26 (2): 612-633.

[19] Kalombo KD. (2016). Evolution des éléments du climat en RDC, Ed. Universitaire Europenne.

[20] Mongeke MM., Ngbolua KN., Bakola RD., Inkoto CL., Elikandani PN., Mowuli CY. (2018). Enquête sur les plantes utilisées en Médicine Traditionnelle par les Bambenga: Pygmées du Secteur de Dongo (Territoire de Kungu, Province du Sud-Ubangi) en République démocratique du Congo. Revue Marocaine des Sciences Agronomiques et Vétérinaires; Vol.n6 pp4.
[21] Ndjouondo GP., Ngene J-P., Ngoule CC., Kidik PM-C., Ndjib RC., Dibong SD., Mpondo ME. (2015). Inventaire et caractérisation des plantes médicinales des sous bassins versants Kambo et Longmayagui (Douala, Cameroun). Journal of Animal \&Plant Sciences, 25 (3): 3898 -3916

[22] Kikufi A., Lejoly J. and Lukoki F. (2017). État actuel de la biodiversité végétale du territoire de Kimvula au sud-ouest de la République Démocratique du Congo International Journal of Innovation and Applied Studies, 19 (4): 929-943.

[23] Ngbolua KN., Mpiana PT., Mudogo V., Ngombe NK., Tshibangu DST., Ekutsu E., Kabena ON., Gbolo BZ \& Muanyishay L. (2014d). Ethno-pharmacological survey and Floristical study of some Medicinal Plants traditionally used to treat infectious and parasitic pathologies in the Democratic Republic of Congo. International Journal of Medicinal Plants 106: 454-467.

[24] Betti JL., Yongo OL., Mbomio DO., Iponga DM \& Ngoye A. (2013). An ethno-botanical and floristical study of medicinal plants among the Baka Pygmies in the periphery of the IpassaBiosphere reserve, Gabon. European Journal of Medicinal Plants 3 (2): 174-205.

[25] Mulwele FN., Ngbolua KN., Masens YD-M and Mpiana PT. (2016). Etudes ethnobotanique et écologique des plantes utilisées dans le traitement de la stérilité à Kenge et ses environs (Province du Kwango, République Démocratique du Congo). nternational Journal of Innovation and Scientific Research 26 (2): 600-611.

[26] Lakouéténé DPB., Ndolngar G., Berké B., Moyen JM., Komba EK., Zinga I., Silla S., Millogo-Rasolodimby J., Vincendeau P., Syssa-Magalé JL., Nacoulma-Ouedrago OG., Laganie R., Badoc A., Chèze C. (2009). Enquête ethnobotanique des plantes utilisées dans le traitement du paludisme à Bangui. Bull. Soc. Pharm. 148: 123 - 138.

[27] Anyinam C. 1995. Ecology and ethnomedicine: exploring links between current environmental crisis and indigenous medical practices. Social Science and Medicine. 4: 321 - 329.

[28] Benkhnigue O., Zidane L., Fadli M., Elyacoubi H., Rochdi A., Douira A. (2011). Étude ethnobotanique des plantes médicinales dans la région de Mechraâ Bel Ksiri (Région du Gharb du Maroc). Acta. Bot. Barc. 53: 191 - 216.

[29] Kahouadji A. (2007). Étude ethnobotanique de la flore médicinale dans la région de Rabat (Maroc occidental) LAZAROA 28:79-93. 